\title{
Studying on a Genetic-Simulation Optimization Algorithm Method for Steel Crane Scheduling Problem
}

\author{
Gao Xiaoqiang ${ }^{1, *}$, Li Pan ${ }^{1}$, Zheng Zhong ${ }^{2}$, Jiang Shenglong ${ }^{2}$, You Xiao \\ ${ }^{1}$ College of Economics and Business Administration, Chongqing University, Chongqing, China \\ ${ }^{2}$ College of Material Science and Engineering, Chongqing University, Chongqing, China
}

\section{Email address:}

xqgao@cqu.edu.cn (Gao Xiaoqiang), lipan@cqu.edu.cn (Li Pan), zhengzh@cqu.edu.cn (Zheng Zhong), jiang_shl@cqu.edu.cn (Jiang Shenglong), youxi@cqu.edu.cn (You Xiao)

${ }^{*}$ Corresponding author

\section{To cite this article:}

Gao Xiaoqiang, Li Pan, Zheng Zhong, Jiang Shenglong, You Xiao. Studying on a Genetic-Simulation Optimization Algorithm Method for Steel Crane Scheduling Problem. Science Innovation. Vol. 4, No. 6, 2016, pp. 283-289. doi: 10.11648/j.si.20160406.17

Received: November 16, 2016; Accepted: December 3, 2016; Published: December 7, 2016

\begin{abstract}
For solving crane scheduling problem in steelmaking-continuous casting process, a hybrid method of integrating genetic algorithm and simulation was formulated to minimize the operation time of tasks by considering full ladles, empty ladles and auxiliary tasks as transportation tasks. The crane selected sequence was designed as chromosome coding. The initial population was generated by employing the crane selection rules in the simulation model, and then evolved optimized by using the genetic operations such as selection, crossover and mutation. The chromosome is evaluated through generating a feasible crane schedule by employing the attribute updating rules and collision eliminating rules in the simulation model. The new crane scheduling could be generated through simulation model based on the new species. Therefore, more excellent individuals will be produced along with the evolutionary process. Thus a better crane scheduling scheme could be obtained finally. In the process of initialization, the high quality initial population was generated in the simulation model. In the iterative process, simulation model also could generate the feasible crane scheduling schemes based on the given cranes selection sequence. To validate this model, experiments were conducted by using the production data in the casting span of a steel plant. The results demonstrate the feasibility and efficiency of this method, which provides a useful tool for crane scheduling in actual production.
\end{abstract}

Keywords: Crane Scheduling, Simulation Model, Genetic Algorithm, Simulation Rules

\section{对面向钢厂天车调度问题的遗传-仿真优化求解方法的研究}

\author{
高小强 ${ }^{1 *}$, 李盼 ${ }^{1}$, 郑忠 ${ }^{2}$, 蒋胜龙 ${ }^{2}$, 游潇 ${ }^{2}$ \\ ${ }^{1}$ 经济与工商管理学院, 重庆大学, 重庆, 中国 \\ ${ }^{2}$ 材料科学与工程学院, 重庆大学, 重庆, 中国
}

邮箱

xqgao@cqu. edu. cn（高小强），1ipan@cqu. edu. cn（李盼）, zhengzh@cqu. edu.cn（郑忠）,

jiang_sh1@cqu. edu.cn (蒋胜龙), youxi@cqu. edu. cn（游潇）

摘要: 针对钢厂天车调度问题, 以重钢包运输、空钢包运输以及突发辅助运输作为天车调度任务, 考虑天车吊运过程 中的空间和时间相关约束条件, 提出了遗传算法与仿真模型混合优化求解方法: 以任务的天车选择序列作为染色体编 码, 使用仿真模型的择车规则产生初始种群, 然后经由选择、交叉和变异等遗传操作产生新种群, 再通过仿真运行的 天车属性更新规则、冲突处理规则产生可行的天车调度方案, 如此经过不断进化迭代获得较优的天车调度方案。在初 始化过程中, 仿真模型使用不同的择车规则产生较高质量的初始种群; 在迭代过程中, 基于给定天车选择序列, 仿真 
模型可快速确定可行天车调度方案。采用某钢厂浇铸跨的生产数据进行实验, 结果表明设计的求解方法能够获得较好 的天车调度方案, 可为实际生产过程中的天车调度提供有效指导。

关键词：天车调度, 仿真模型, 遗传算法, 仿真规则

\section{1. 引言}

在钢厂生产车间中, 天车是最重要的运输工具, 合理、 有效的天车调度方案对于提高物流效率和钢厂整体效益 至关重要 $[1]$ 。钢厂天车调度问题是指在满足天车运行空 间约束、任务时间约束的条件下，为任务分配天车，并确 定每个任务的实际运行时间, 从而保证生产平稳、有序地 进行。在钢厂的作业跨中, 由于同一时间段内需要天车吊 运的任务较多, 且路线之间相互交叉, 而多台天车只能在 同一条轨道上运行（如图1所示），这种受时间、空间约 束条件限制、多机多任务的特点, 使得其难以求解, 是典 型的NP难题 [2]。

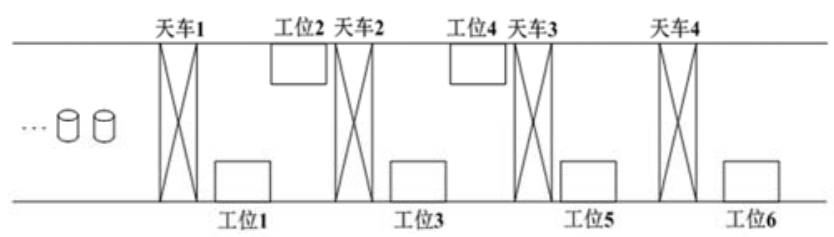

图1 作业跨示意图。

目前国内外对天车调度的研究集中在码头集装箱的 调度问题上 [3 5], 而关于钢厂的天车调度的研究比较少。 在研究问题方面, 现有研究大多以只以重钢包为吊运对象, 忽略了空钢包运输 $[6,9]$, 且只针对已确定的任务, 不能 实时根据任务制定天车调度方案 [8 12]。而在实际生产中 经常会突发一些需要天车吊运的辅助任务, 导致了原制定 好的天车调度方案不再可行, 需要重新制定。在研究方法 上, 目前对钢厂的天车调度的研究方法主要包括数学规划 模型 $[6 \sim 8,10]$ 、仿真模型 $[9,11 \sim 14]$ 等建模方法, 以及智 能算法 $[8,10-11]$ 等求解方法。数学规划法建立的天车调 度模型的结构化特征明显, 难以反映调度过程的各种随机 性和不确定性; 而仿真模型能反映出系统内各要素的关系, 但在优化性能方面不足; 各种智能求解算法需结合问题的 特点进行改进以更好发挥优化作用。

针对以上问题, 本研究以浇铸跨为背景, 以重钢包运 输、空钢包运输、突发的辅助运输为吊运任务, 以所有任 务总运行时间为优化目标, 考虑时空约束条件, 提出一种 遗传算法与仿真模型混合优化求解方法。按任务序列排序 的天车选择序列作为染色体编码, 通过多次运行仿真模型 的任务择车规则, 得到的多个天车选择序列作为遗传算法 的初始种群, 经过遗传进化算子迭代优化。遗传算法产生 的新种群再次通过仿真运行可得到对应的天车调度方案, 如此循环往复得到较优天车调度方案。通过以某钢厂的浇 铸跨的生产数据为测试数据进行测试, 以说明算法的可行 性和有效性。

\section{2. 遗传算法与仿真模型混合优化求解方法设计}

当辅助任务发生时, 原天车调度方案不再可行, 需要 重新制定。统计此时正在被吊运的任务、未发生的任务以 及辅助任务, 按照最早起吊时间先后顺序排列成任务序列, 包含每个任务的最早起吊、最晚卸载时间, 起吊、卸载位 置。天车选择序列对应于任务序列, 是每个任务所选择的 天车的排序, 以天车选择序列作为一个染色体。利用遗传 算法与仿真模型相结合的优化求解方法求解天车调度问 题就是为所有任务分配天车, 确定天车选择序列及每个任 务的实际起吊、卸载时间, 即得到天车调度方案, 遗传算 法与仿真模型混合优化求解方法流程如图2所示。

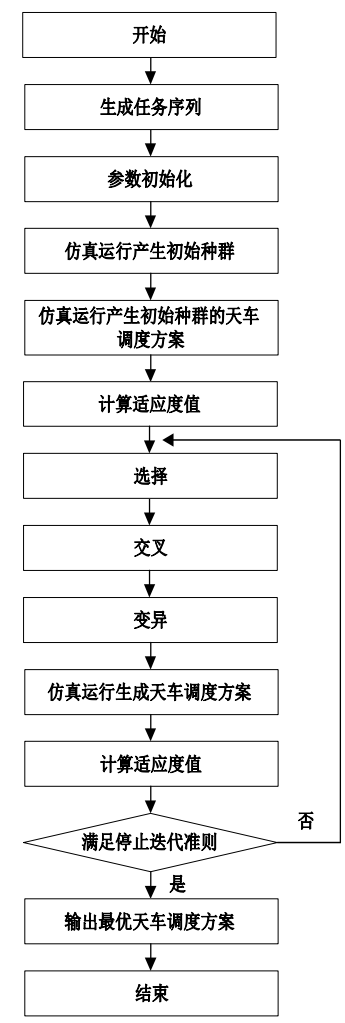

图2 遗传算法与仿真模型混合优化求解方法流程图。

在图2中, 遗传算法的初始种群是通过仿真模型产生, 任务序列通过仿真模型的任务择车规则可生成对应的天 车选择序列, 再经过仿真运模型的天车属性更新规则、冲 突处理规则生成对应于天车选择序列的天车调度方案, 如 此多次仿真即可生成多个天车选择序列和对应的多个天 车调度方案。天车调度方案是天车选择序列的具体展现, 用以计算适应度值。而天车选择序列作为遗传算法的初始 种群通过选择、交叉、变异进行迭代优化。产生的新种群 
通过仿真运行可得到对应的多个天车调度方案。通过不断 的迭代和仿真, 最终输出较优的天车调度方案。

\section{1. 仿真模型}

天车调度仿真模型包含任务和天车两个对象, 任务是 指由生产作业计划得到的需要天车吊运的任务以及实际 生产中临时发生的辅助任务。在仿真模型中通过天车属性 的变化来表征其吊运任务的过程。为便于表达仿真模型, 引入符号体系如下:

任务集: $i \in\{1,2, \ldots, M\}$ ；

天车集: $j \in\{1,2, \ldots, N\}$;

$t$ : 天车运行的时间点用离散化表达, 以秒为单位; $t=T_{S}, \cdots, T_{E}$ (调度时间域)；

$t_{i s}$ : 任务 $i$ 的最早起吊时间;

$t_{i e}$ : 任务 $i$ 的最晚卸载时间;

$d_{i s}:$ 任务 $i$ 的起吊位置;

$d_{i e}$ : 任务 $i$ 的卸载位置;

仿真模型的数学表达式如下:

$$
\begin{gathered}
\min f_{1}=\min \sum_{i=1}^{M}\left(t_{i e}^{\prime}-t_{i s}^{\prime}\right) \\
t_{i s}^{\prime} \geq t_{i s}, \forall i \in\{1,2, \ldots, M\} \\
t_{i e}^{\prime} \leq t_{i e}, \forall i \in\{1,2, \ldots, M\} \\
d_{j}^{t}-d_{j-1}^{t} \geq \delta, \forall j \in\{2, \ldots, N\}, t \in\{1,2, \ldots, T\}
\end{gathered}
$$

式 (1) 表示为仿真模型的优化目标, 即所有任务的 总运行时间最小, $t_{i s}^{\prime} 、 t_{i e}^{\prime}$ 分别为任务 $i$ 的实际起吊、实 际卸载时间; 式 (2) 表示任务的实际起吊时间不能小于 最早起吊时间; 式 (3) 表示任务的实际卸载时间不能大 于最晚卸载时间; 式 (4) 表示任意时刻相邻两台天车间 的距离不小于最小安全距离 $\delta$ 。

天车调度问题的特点包括: 1) 作业跨中的天车只能 在同一条轨道上运行, 天车之间相对位置固定, 不能彼 此跨越；2）一个任务只能选择由一台天车吊运；3）一台 天车同一时刻只能吊运一个任务。

在利用仿真模型描述天车调度问题时, 不仅要能反 映上述问题特点, 而且还要描述任务、天车的属性以及 调度规则。任务属性包含: 最早起吊、最晚卸载时间, 实际起吊、卸载时间, 起吊、卸载位置, 任务优先级, 任务类别。天车属性包含: 天车正在吊运的任务, 位置, 状态, 所处阶段, 目标位置, 是否被动。任务属性是已 知输入, 天车属性是通过仿真运行需要求解的结果。

仿真模型是在一定的规则指导下运行, 第一次迭代 时, 需要通过任务择车规则选择天车, 再通过天车属性 的更新来表征任务的吊运过程, 在此过程中, 由于时空 约束的限制, 相邻天车间可能会发生冲突, 因此通过设 计冲突预测规则, 提前预测可能发生的冲突并进行消解。 后续迭代时, 由于遗传算法生成的新种群, 即天车选择
序列已经给定, 不再需要择车, 按照选择的天车更新天 车属性即可生成对应的天车调度方案。

\section{1. 1 . 任务择车规则}

任务择车规则用于为任务分配天车, 在第一次迭代时, 通过任务择车规则为任务序列中每个任务都分配天车, 产 生一个天车选择序列。通过多次仿真运行, 可产生多个天 车选择序列, 即作为遗传算法的初始种群。任务择车规则 是根据任务发生时刻, 作业跨中各天车的状态和位置及与 任务起吊、卸载位置的相对位置关系选择适当的天车, 步 骤如下:

1) 确定备选天车集合。以任务 $i$ 为例, $t_{i s}$ 时刻空载状 态的天车可作为备选天车, 而对于 $t_{i s}$ 时刻负载状态 的天车需要判断其是否能在最晚卸载时间之前完成 任务 $i$ 的吊运, 若能满足也可作为备选天车。

2) 分配天车优先级。根据天车位置与任务 $i$ 起吊、卸载 位置的相对位置关系及其移动方向确定各天车的优 先级, 依据任务 $i$ 的起吊卸载位置将作业跨划分为 3 块： $a$. 位于起吊和卸载位置中间的备选天车优先级 为 $3 ; b$. 不处于起吊和卸载位置中间的备选天车, 靠 近起吊位置的备选天车优先级为 $2 ; c$. 不处于起吊和 卸载位置中间的备选天车, 靠近卸载位置的备选天 车优先级为 1 。

3) 选择天车。在优先级最高的备选天车集合中随机选 择一台天车。

\section{1. 2. 天车属性更新规则}

天车状态包含负载和空载状态。天车吊运任务时, 为 负载状态, 天车任务属性为吊运的任务标号。当天车完成 卸载时, 为空载状态, 任务属性为 0 。天车吊运任务的过 程可划分为起吊阶段和卸载阶段, 起吊阶段为天车从当前 位置向起吊位置移动, 并完成起吊的过程, 在此阶段天车 的目标位置为起吊位置; 卸载阶段为天车吊运任务向卸载 位置移动, 并完成卸载的过程, 在此阶段天车的目标位置 为卸载位置。而对于空载状态天车, 其目标位置可看做当 前位置。

在吊运过程中天车位置变化与天车的移动方向有关。 若天车的目标位置大于天车当前位置, 天车正向移动, 位 置 $d_{j}^{t+1}=d_{j}^{t}+v$, 反之反向移动, 位置 $d_{j}^{t+1}=d_{j}^{t}-v$, 其中 $v$ 为天车移动速率。

天车吊运任务过程中, 各属性的更新如图3所示。

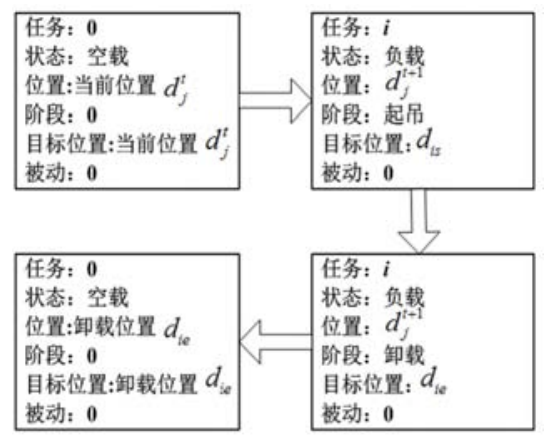

图3 天车属性更新规则。 


\section{1. 3. 冲突处理规则}

在作业跨中, 所有天车只能在同一条轨道上运行, 当 相邻天车的间距达到 $\delta$ 时就发生了冲突, 为了消解冲突, 其中一台天车必然要跟车行驶, 从而降低了天车吊运效率, 甚至打乱整个生产计划。为此提出一种冲突预测规则和基 于任务优先级的冲突消解规则来处理冲突。

(1) 冲突预测规则

由于天车的空载位置不同导致两个阶段中天车的移 动方向可能会不同, 设定在每阶段的开始时刻通过判断天 车所处的阶段, 并与其他天车的目标位置进行比较来判断 是否会发生冲突。

以天车 $j$ 吊运任务 $i$ 为例, $o d_{j}^{t}$ 为天车 $j$ 当前阶段的目 标位置, 在起吊或卸载阶段的起始时刻, 判断是否存在天 车 $k$ 使 得 $o d_{j}^{t}+(k-j) \delta>o d_{k}^{t}(k=j+1, \ldots, N)$ 或 $o d_{j}^{t}-(j-k) \delta<o d_{k}^{t}(k=1, \ldots, j-1)$ 成立, 若存在即天车 $j$ 与 天车 $k$ 会发生冲突。

(2) 冲突消解规则

当预测发生冲突后, 采用基于任务优先级的方法进行 冲突消解。设重钢包任务数为 $M_{1}$, 空钢包任务数为 $M_{2}$, 优先级规则设计如下：1）对于重钢包任务, 首先将其按 最晚卸载时间 $t_{i e}$ 的先后顺序排序, $t_{i e}$ 最小的优先级最高 为 $M_{1}+M_{2}$, 后续依次减 $1, t_{i e}$ 最大的优先级最低为 $M_{2}+1$ 。 2) 对于空钢包任务, 首先将其按最早起吊时间 $t_{i s}$ 的先后 顺序排序, $t_{i s}$ 最小的优先级最高为 $M_{2}$, 后续依次减 $1, t_{i s}$ 最大的优先级最低为 1 。3) 对于辅助任务, 需要根据实际 生产情况确定其优先级。4) 天车空载状态, 吊运虚任务, 其优先级为 0 。5) 处于正在起吊或卸载的任务优先级最高 为 $M_{\infty}$ （极大值）, 完成后, 任务优先级再恢复至原值。

当预测天车 $j$ 与 $k$ 发生冲突, 比较二者吊运的任务优 先级, 若天车 $k$ 吊运的任务优先级较低, 则天车 $j$ 的属性 按照图3进行更新, 而天车 $k$ 的属性更新规则如图 4 , 其中 空载状态属性更新规则如分图 (a), 负载状态属性更新规 则如分图 (b) 所示。

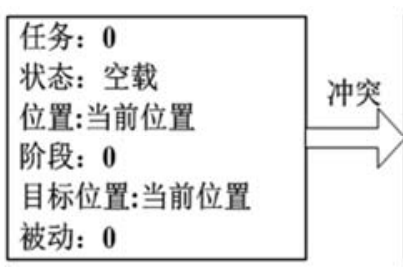

(a)

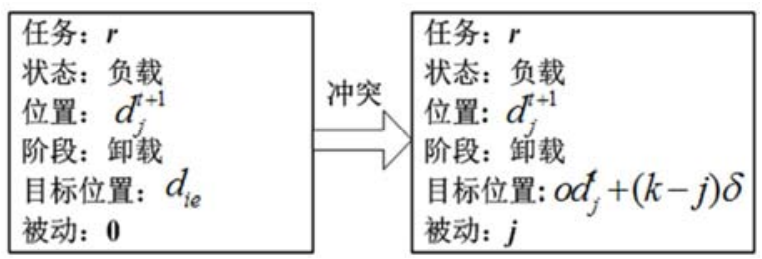

(b)

\begin{tabular}{|l|}
\hline 任务: $\mathbf{0}$ \\
状态: 空载 \\
位置: $d_{k}^{t+1}$ \\
阶段: 0 \\
目标位置: $o d_{j}^{t}+(k-j) \delta$ \\
被动: $j$
\end{tabular}

(a) b)
图4 冲突时低任务优先级天车属性更新规则。

\section{1. 4. 仿真运行过程}

单次仿真运行的过程可以划分为 4 部分: (1)仿真时钟 初始化与参数初始化, 设置仿真时钟为第一个任务的最早 起吊时间, 设置任务标号 $i=1$, 天车标号 $j=1$ 。(2)任务 循环, 逐一判断当前时刻发生的任务, 并为其分配天车。 (3)天车循环。逐一判断天车所处的阶段, 在各阶段起始时 刻预测冲突并进行冲突消解, 再根据天车 $t$ 时刻各属性推 演 $t+1$ 时刻的各属性。(4)仿真时钟推进。通过仿真运行可 为任务分配天车, 得到每个任务的实际吊运时间以及天车 运行过程。单次仿真结束时, 可得到按任务序列排序的对 应的天车选择序列及天车调度方案。仿真运行过程详细流 程图如图5所示。

\section{2. 遗传算法}

仿真运行输出的结果需要通过遗传算法迭代优化, 设 计遗传算法如下:

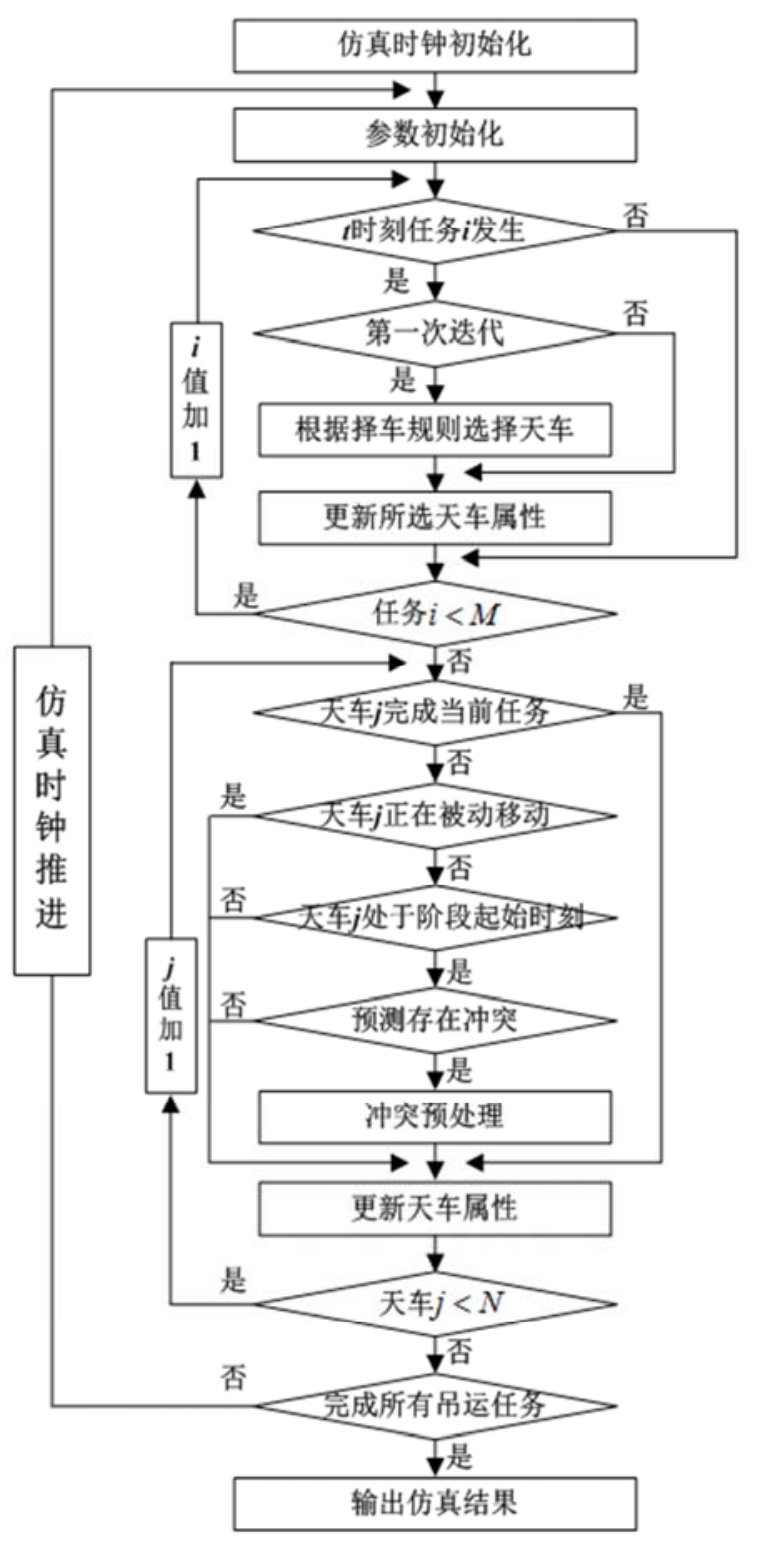

图5 仿真流程图。 
（1）编码设计。采用整数编码法, 染色体由对应任务序 列的天车序列组成, 染色体中每个基因值代表对应 任务选择的天车，基因排列顺序就是对应任务排列 的顺序。初始种群利用仿真模型的择车规则生成, 提高初始染色体的可行性。而经过进化迭代产生的 新种群就是一组已经确定的天车选择序列, 通过仿 真运行时, 不再需要择车, 直接按照选择的天车运 行即可。

(2) 适应度函数设计。仿真模型的优化目标为所有任务 总运行时间最小, 通过仿真运行得到天车调度方案, 以此设计适应度函数如式 (5)。

$$
\operatorname{Fit}(j)=\left(T_{E}-T_{S}\right)-f(j)
$$

式 (5) 中 $T_{E}-T_{S}$ 为调度时间域, 通过计算适应度值, 使得种群中目标函数值最小的染色体, 其适应度值最大, 遗传到下一代的几率更高。

（3）进化算子设计。选择算子采用最优保存法和锦标赛 选择法, 首先将适应度最大的染色体予以保存, 再
在当前种群中随机选择两个个体, 将较优的个体复 制到下一代。交叉算子采用两点交叉策略。步骤为: 按照交叉概率选择两个个体, 在个体编码串中随机 设置了两个交叉点, 然后再进行部分基因交换。变 异算子采用单点变异法, 按照变异概率选择一个个 体, 随机选定变异位置, 随机选择一台天车, 将基 因值改变为相应天车编码。

（4）停止迭代准则: 当迭代次数达到 500 代, 且连续 10 代种群的最大适应度值相同，停止迭代。

\section{3. 实验设计及结果分析}

某钢厂的浇铸作业跨布局如图6所示, 原任务序列及 通过求解得到原天车调度方案如表1所示, 天车的运行过 程如图7所示。设计临时发生的需要天车吊运的辅助任务 来进行仿真实验, 利用遗传算法与仿真模型相结合的优化 求解方法来求解天车调度方案, 结果如表 2 所示, 相应的 天车的运行过程如图8所示。

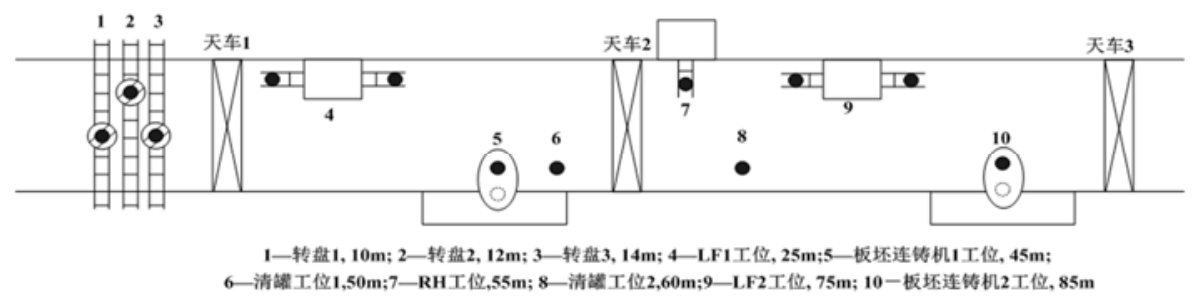

图6 浇铸跨布局图。

表1 初始天车调度方案。

\begin{tabular}{|c|c|c|c|c|c|c|c|c|c|c|c|c|c|c|c|}
\hline 序号 & $\begin{array}{l}\text { 起始 } \\
\text { 工位 }\end{array}$ & $\begin{array}{l}\text { 卸载 } \\
\text { 工位 }\end{array}$ & $\begin{array}{l}\text { 最早起吊 } \\
\text { 时间 }\end{array}$ & $\begin{array}{l}\text { 最晚卸载 } \\
\text { 时间 }\end{array}$ & 天车 & $\begin{array}{l}\text { 实际起 } \\
\text { 吊时间 }\end{array}$ & $\begin{array}{l}\text { 实际卸载 } \\
\text { 时间 }\end{array}$ & 序号 & $\begin{array}{l}\text { 起始 } \\
\text { 工位 }\end{array}$ & $\begin{array}{l}\text { 卸载 } \\
\text { 工位 }\end{array}$ & $\begin{array}{l}\text { 最早起吊 } \\
\text { 时间 }\end{array}$ & $\begin{array}{l}\text { 最晚卸载 } \\
\text { 时间 }\end{array}$ & 天车 & $\begin{array}{l}\text { 实际起吊 } \\
\text { 时间 }\end{array}$ & $\begin{array}{l}\text { 实际卸 } \\
\text { 载时间 }\end{array}$ \\
\hline 1 & 转盘3 & LF2 & $0: 31: 04$ & $0: 46: 10$ & 3 & $0: 31: 55$ & $0: 34: 56$ & 12 & 铸机2 & 清罐2 & $2: 38: 44$ & $4: 00: 00$ & 2 & $2: 38: 44$ & $2: 41: 09$ \\
\hline 2 & 转盘1 & LF1 & $0: 59: 08$ & $1: 13: 56$ & 2 & $0: 59: 09$ & $1: 01: 24$ & 13 & 铸机1 & 清罐1 & $2: 43: 14$ & $4: 00: 00$ & 2 & $2: 43: 29$ & $2: 45: 34$ \\
\hline 3 & 转盘3 & LF2 & $1: 20: 01$ & $1: 35: 07$ & 2 & $1: 20: 12$ & $1: 23: 13$ & 14 & LF2 & 铸机2 & $2: 48: 38$ & $3: 21: 14$ & 2 & $2: 49: 03$ & $2: 51: 13$ \\
\hline 4 & LF2 & 铸机2 & $1: 29: 34$ & $1: 53: 14$ & 2 & $1: 29: 34$ & $1: 31: 44$ & 15 & 转盘1 & LF1 & $3: 03: 52$ & $3: 19: 02$ & 3 & $3: 05: 10$ & $3: 07: 25$ \\
\hline 5 & 转盘2 & LF1 & $1: 32: 12$ & $1: 46: 42$ & 1 & $1: 32: 19$ & $1: 34: 32$ & 16 & 转盘2 & LF2 & $3: 07: 42$ & $3: 32: 45$ & 3 & $3: 07: 55$ & $3: 10: 58$ \\
\hline 7 & 转盘1 & LF2 & $1: 50: 16$ & $2: 05: 22$ & 1 & $1: 50: 51$ & $1: 53: 56$ & 18 & 铸机2 & 清罐2 & $3: 20: 44$ & $4: 00: 00$ & 3 & $3: 20: 54$ & $3: 23: 19$ \\
\hline 8 & LF2 & 铸机1 & $2: 05: 12$ & $2: 44: 04$ & 1 & $2: 05: 12$ & $2: 07: 42$ & 19 & 铸机1 & 清罐1 & $3: 26: 12$ & $4: 00: 00$ & 2 & $3: 26: 12$ & $3: 28: 17$ \\
\hline 9 & 转盘2 & LF1 & $2: 22: 45$ & $2: 37: 20$ & 2 & $2: 23: 58$ & $2: 26: 11$ & 20 & LF2 & $\mathrm{RH}$ & $3: 29: 35$ & $3: 32: 10$ & 3 & $3: 29: 50$ & $3: 32: 10$ \\
\hline 10 & LF1 & 铸机2 & $2: 29: 56$ & $2: 39: 14$ & 2 & $2: 29: 56$ & $2: 32: 56$ & 21 & 转盘3 & LF1 & $3: 46: 20$ & $4: 00: 44$ & 1 & $3: 46: 30$ & $3: 48: 41$ \\
\hline 11 & 转盘3 & LF2 & $2: 33: 42$ & $2: 48: 48$ & 1 & $2: 33: 47$ & $2: 36: 48$ & 22 & LF1 & 铸机1 & $3: 56: 32$ & $4: 09: 14$ & 1 & $3: 56: 32$ & $3: 58: 52$ \\
\hline
\end{tabular}

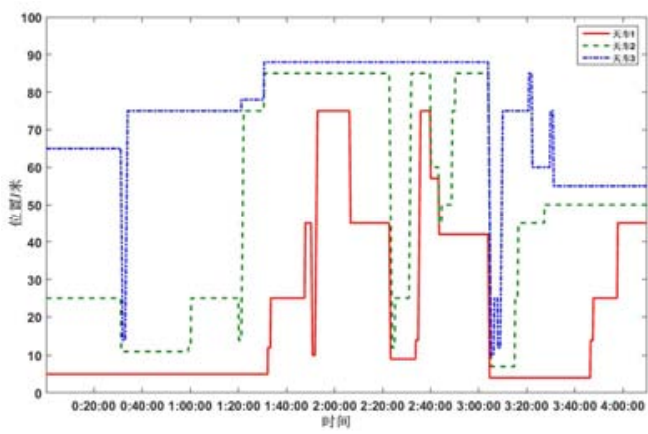

图7 天车运行位置图。

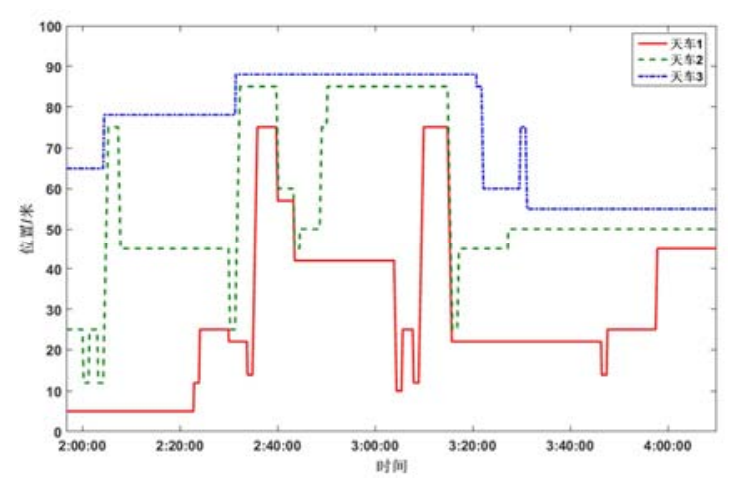

图8 天车运行位置图。 
表1中原任务序列中共有 22 项任务，其中重钢包共 18 项，空钢包4项。由表1可看出，每个任务都能在预计吊运 时间内完成。图7中显示了优化方案中三台天车运行过程,
可看出天车之间的轨迹无交叉, 相对位置保持不变, 且相 邻天车的间距均不小于安全距离, 是合理可行的调度方案。

表2 调整后天车调度方案。

\begin{tabular}{|c|c|c|c|c|c|c|c|c|c|c|c|c|c|c|c|}
\hline 序号 & $\begin{array}{l}\text { 起始 } \\
\text { 工位 }\end{array}$ & $\begin{array}{l}\text { 卸载 } \\
\text { 工位 }\end{array}$ & $\begin{array}{l}\text { 最早起吊 } \\
\text { 时间 }\end{array}$ & $\begin{array}{l}\text { 最晚卸载 } \\
\text { 时间 }\end{array}$ & 天车 & $\begin{array}{l}\text { 实际起 } \\
\text { 吊时间 }\end{array}$ & $\begin{array}{l}\text { 实际卸载 } \\
\text { 时间 }\end{array}$ & 序号 & $\begin{array}{l}\text { 起始 } \\
\text { 工位 }\end{array}$ & $\begin{array}{l}\text { 卸载 } \\
\text { 工位 }\end{array}$ & $\begin{array}{l}\text { 最早起吊 } \\
\text { 时间 }\end{array}$ & $\begin{array}{l}\text { 最晚卸载 } \\
\text { 时间 }\end{array}$ & 天车 & $\begin{array}{l}\text { 实际起吊 } \\
\text { 时间 }\end{array}$ & $\begin{array}{l}\text { 实际卸载 } \\
\text { 时间 }\end{array}$ \\
\hline 1 & 转盘2 & LF1 & $2: 00: 00$ & $2: 22: 45$ & 2 & $2: 00: 13$ & $2: 02: 26$ & 15 & 转盘1 & LF1 & $3: 03: 52$ & $3: 19: 02$ & 3 & $3: 05: 10$ & $3: 07: 25$ \\
\hline 2 & 转盘2 & LF2 & 2:03:00 & $2: 33: 42$ & 2 & $2: 03: 13$ & $2: 06: 16$ & 16 & 转盘2 & LF2 & $3: 07: 42$ & $3: 32: 45$ & 3 & $3: 07: 55$ & $3: 10: 58$ \\
\hline 8 & LF2 & 铸机1 & $2: 05: 12$ & $2: 44: 04$ & 1 & $2: 05: 12$ & $2: 07: 42$ & 17 & LF1 & 铸机1 & $3: 14: 50$ & $3: 28: 32$ & 2 & $3: 15: 08$ & $3: 17: 28$ \\
\hline 9 & 转盘2 & LF1 & $2: 22: 45$ & $2: 37: 20$ & 2 & $2: 23: 58$ & $2: 26: 11$ & 18 & 铸机2 & 清罐2 & $3: 20: 44$ & $4: 00: 00$ & 3 & $3: 20: 54$ & $3: 23: 19$ \\
\hline 10 & LF1 & 铸机2 & $2: 29: 56$ & $2: 39: 14$ & 2 & $2: 29: 56$ & $2: 32: 56$ & 19 & 铸机1 & 清罐1 & $3: 26: 12$ & $4: 00: 00$ & 2 & $3: 26: 12$ & $3: 28: 17$ \\
\hline 11 & 转盘3 & LF2 & $2: 33: 42$ & $2: 48: 48$ & 1 & $2: 33: 47$ & $2: 36: 48$ & 20 & LF2 & $\mathrm{RH}$ & $3: 29: 35$ & $3: 32: 10$ & 3 & $3: 29: 50$ & $3: 32: 10$ \\
\hline 12 & 铸机 2 & 清罐2 & $2: 38: 44$ & $4: 00: 00$ & 2 & $2: 38: 44$ & $2: 41: 09$ & 21 & 转盘3 & LF1 & $3: 46: 20$ & $4: 00: 44$ & 1 & $3: 46: 30$ & $3: 48: 41$ \\
\hline 13 & 铸机1 & 清罐1 & $2: 43: 14$ & $4: 00: 00$ & 2 & $2: 43: 29$ & $2: 45: 34$ & 22 & LF1 & 铸机1 & $3: 56: 32$ & $4: 09: 14$ & 1 & $3: 56: 32$ & $3: 58: 52$ \\
\hline 14 & LF2 & 铸机2 & $2: 48: 38$ & $3: 21: 14$ & 2 & $2: 49: 03$ & $2: 51: 13$ & & & & & & & & \\
\hline
\end{tabular}

由表2可以看出, 调整后的天车调度方案不仅顺利完 成了辅助任务的吊运, 也在时间限制范围内完成了各任务 的吊运。图8显示了相应的三台天车的运行过程, 三台天 车之间轨迹无交叉, 且能在每个阶段的起始时刻准确预测 到天车冲突, 低优先级的天车也能立即移动至其目标位置, 有效的避免了碰撞的发生。因此说明调整后天车调度方案 满足了任务时间约束、天车运行的空间约束, 是可行有效 的天车调度方案。

\section{4. 结论}

针对炼钢厂浇铸跨天车调度问题，以重钢包运输、空钢 包运输、突发辅助运输为调度任务, 以所有任务总运行时间 为优化目标, 考虑时空约束条件, 设计了遗传算法与仿真模 型混合优化求解方法, 提出了基于任务特点的任务择车规则、 天车属性更新规则、冲突处理规则。设计以天车选择序列为 染色体, 通过仿真模型的任务择车规则为各任务分配天车, 产生初始种群, 然后通过遗传进化进行迭代寻优。新种群经 过仿真运行生成天车调度方案, 如此循环往复得到较优天车 调度方案。通过对某钢厂的浇铸跨的生产数据进行测试, 得 到初始天车调度方案。在初始天车调度方案基础上设置了随 机发生的辅助任务, 通过求解得到调整后的天车调度方案, 说明了混合优化求解方法的可行性和有效性。

\section{致谢}

本文为国家自然科学基金资助项目《炼钢厂生产实时 调度的动态网络建模及调度策略优化方法》（51474044） 的阶段性成果之一。

\section{参考文献}

[1] 刘青, 田乃媛, 王英群, 等. 天车调度在优化钢厂物流管制中 的重要作用 $[J]$. 北京科技大学学报, 1998(1) : 36-40。
[2] Jalilvand-Nejad A, Fattahi P. A mathematical model and genetic algorithm to cyclic flexible job shop scheduling problem $[\mathrm{J}]$. Journal of Intelligent Manufacturing, 2013, 26 (6) : 1-14.

[3] Ng W C. Crane scheduling in container yards with inter-crane interference $[\mathrm{J}]$. European Journal of Operational Research, 2005, 164 (1) : 64-78.

[4] Li W, Wu Y, Goh M. Discrete Time Model and Algorithms for Container Yard Crane Scheduling [J]. European Journal of Operational Research, 2009, 198 (1): 165-172.

[5] Wu Y, Li W, Petering M E H, et al. Scheduling Multiple Yard Cranes with Crane Interference and Safety Distance Requirement [J]. Transportation Science, 2015, 49 (4).

[6] Tanizaki T, Tamura T, Sakai H, et al. A heuristic scheduling algorithm for steel making process with crane handling $[\mathrm{J}]$. Journal of the Operations Research Society of Japan, 2006, 3 (3) : 188-201.

[7] De 1 Vecchio C, Barbarisi 0, Parisio A. Hybrid Model for Crane Scheduling [J]. 2008.

[8] Liu P, Tang L X. The refining scheduling problem with crane non-collision constraint in steelmaking process $[\mathrm{C}] / /$ Automation and Logistics, 2008. ICAL 2008. IEEE International Conference on. IEEE, 2008: $536-541$.

[9] 何明, 唐秋华, 王盛龙. 炼钢-连铸天车调度规则设计与评价 $[J]$. 机械设计与制造, 2012, (9) :257-259。

[10] 王旭, 刘诗新, 王佳. 求解具有时空约束的天车调度问题 Memetic 算法 $[J]$. 东北大学学报（自然科学 版) , 2014, 35 (2) : 190-194。

[11] 郑忠, 周超, 陈开. 基于免疫遗传算法的车间天车调度仿真 模型 [J]. 系统工程理论与实践, 2013, 33 (1) : 223-229。 
[12] 刘设, 王世杰, 藏鹏飞, 等. 重型机械加工车间天车调度问题 过程仿真与优化 $[J]$. 机械设计与制造, 2016 (8)。

[13] 朱道飞, 王华, 王建军, 等. 基于Petri网和UML的钢厂天车调 度系统仿真 $[\mathrm{J}]$. 昆明理工大学学报 (自然科学 版), 2013, 38(3):5-11。
[14] 赵宁, 杜彦华, 董绍华, 等. 基于循环仿真的钢铁板坏库天车 作业 优 化 [J]. 系统工程理 论与实 践, 2012, 32 (12) :2825-2830。 